\title{
Videogrammetric Model Deformation Measurement Technique for Wind Tunnel Applications (Invited)
}

\author{
Danny A. Barrows* \\ NASA, Langley Research Center, Hampton, Virginia 23681
}

\begin{abstract}
Videogrammetric measurement technique developments at NASA Langley were driven largely by the need to quantify model deformation at the National Transonic Facility (NTF). This paper summarizes recent wind tunnel applications and issues at the NTF and other NASA Langley facilities including the Transonic Dynamics Tunnel, 31-Inch Mach 10 Tunnel, 8-Ft high Temperature Tunnel, and the 20-Ft Vertical Spin Tunnel. In addition, several adaptations of wind tunnel techniques to non-wind tunnel applications are summarized. These applications include wing deformation measurements on vehicles in flight, determining aerodynamic loads based on optical elastic deformation measurements, measurements on ultralightweight and inflatable space structures, and the use of an object-to-image plane scaling technique to support NASA's Space Exploration program.
\end{abstract}

\section{Introduction}

The measurement of wing bending and twist under aerodynamic load has received considerable interest in the past. Experimental methods applied to wind tunnel models to measure aeroelastic deformations, provide data that can be compared with computational fluid dynamics (CFD) predictions to help validate design geometries. These measurements can also be used to calibrate and validate computational methods such as the finite element method (FEM) to ensure accurate calculations. These assessments are very useful to aircraft designers because acquired wind tunnel data and computational predictions based upon rigid body assumptions do not always agree, thus adversely affecting the outcome of the overall design process. Several techniques have been successfully used for the measurement of wing deformation and twist in a variety of wind tunnels. These techniques include photogrammetry (including passive and active targeting) and projection moiré interferometry (PMI).

The Videogrammetric Model Deformation (VMD) measurement technique ${ }^{1}$ based on single camera, single view photogrammetry and digital image processing of passive targets is the most widely used technique for deformation measurements at NASA Langley. However PMI, a non-intrusive, optical measurement technique that provides spatially continuous measurements of objects ${ }^{2}$ can have advantages for certain applications such as micro-aerial vehicles. ${ }^{3}$ With PMI, images of evenly spaced projected grid lines capture model surface shape and deformation under aerodynamically loaded conditions. The technique offers complete global, full-field measurement coverage but may not be as accurate as videogrammetric techniques and is less suited for real-time applications. In some cases PMI and VMD can be complimentary. Commercially available photogrammetric-based measurement systems that utilize active targets (LEDs) are not normally well suited for aerodynamic wind tunnel testing because of the intrusive nature of the active targets. An increase in fabrication costs is needed to properly affix these targets to a wing for wind tunnel testing. This generally means imbedding the targets into the wing's surface to provide flush mounting and to bury all of the connecting wires. However, photogrammetric-based measurement systems with active targets offer quick data reduction turnaround times and increased accuracy when compared to using passive targets. ${ }^{4}$

*Aerospace Engineer, Advanced Sensing and Optical Measurement Branch, NASA, Langley Research Center/Mail Stop 493, Senior Member AIAA 


\section{Description of Video Photogrammetry}

A VMD measurement system ${ }^{5}$ developed by NASA employs photogrammetry, coupled with video image acquisition, to make a variety of wind tunnel measurements. A typical measurement system includes a PC, an image acquisition frame grabber board, a video-rate charged coupled device (CCD) camera(s), and an automated near real-time image processing architecture. 2-D or 3-D static and dynamic aeroelastic spatial measurements can be obtained using passive retro-reflective tape or painted-on targets. Typically a singlecamera, single-view measurement approach is used to determine two of the three $(X Y Z)$ unknown coordinates in a Cartesian coordinate system if the measurement geometry allows; otherwise a multicamera measurement approach is needed to resolve all three of the unknown coordinates. The single camera technique requires one of the three target coordinates be known in order to solve for the other two unknowns. Errors in a single camera solution depend on how well the "constrained" coordinate is defined. When using two or more cameras for a 3-D photogrammetric solution (solving for all three target coordinates) two equations are obtained for each camera so that a least squares solution is possible for the over-determined set of equations.

\section{A. Photogrammetric Collinearity Equations}

The most fundamental and important data reduction equations in photogrammetry are the collinearity equations (1). They define a relationship between the 3-D spatial coordinates of an object $(X, Y, Z)$ and the corresponding 2-D spatial coordinates for an image $(x, y)$ of that object.

$$
\begin{aligned}
& x=x_{p}-c\left[\frac{m_{11}\left(X-X_{c}\right)+m_{12}\left(Y-Y_{c}\right)+m_{13}\left(Z-Z_{c}\right)}{m_{31}\left(X-X_{c}\right)+m_{32}\left(Y-Y_{c}\right)+m_{33}\left(Z-Z_{c}\right)}\right] \\
& y=y_{p}-c\left[\frac{m_{21}\left(X-X_{c}\right)+m_{22}\left(Y-Y_{c}\right)+m_{23}\left(Z-Z_{c}\right)}{m_{31}\left(X-X_{c}\right)+m_{32}\left(Y-Y_{c}\right)+m_{33}\left(Z-Z_{c}\right)}\right]
\end{aligned}
$$

Where $m_{n}$ are the 9 elements of the rotation matrix

$$
\begin{aligned}
& m_{11}=\cos \phi \cos \kappa \\
& m_{12}=\sin \omega \sin \phi \cos \kappa+\cos \omega \sin \kappa \\
& m_{13}=-\cos \omega \sin \phi \cos \kappa+\sin \omega \sin \kappa \\
& m_{21}=-\cos \phi \sin \kappa \\
& m_{22}=-\sin \omega \sin \phi \sin \kappa+\cos \omega \cos \kappa \\
& m_{23}=\cos \omega \sin \phi \sin \kappa+\sin \omega \cos \kappa \\
& m_{31}=\sin \phi \\
& m_{32}=-\sin \omega \cos \phi \\
& m_{33}=\cos \omega \cos \phi
\end{aligned}
$$

The collinearity equations represent perfect imagery in which the object point, perspective center, and image point lie on a straight line. The terms $x_{p}$ and $y_{p}$ define the photogrammetric principal point location, where the optical axis of the camera intersects the image plane. The principal distance $c$ is the image distance and equals the focal length of a camera when the camera is focused at infinity. The angular rotations around a camera's axis, defined by the Euler angles $(\omega, \phi, \kappa)$, define the image plane (camera sensor) relation to a chosen coordinate system of interest.

\section{B. Camera Calibration}

Internal and external camera orientation parameters must be calculated before any reasonable photogrammetric solutions can be obtained. This allows conversion from pixel coordinates to corrected image plane coordinates. Internal orientation camera parameters $\left(c, x_{p}, y_{p}, K_{1}, K_{2}, P_{1}, P_{2}, S_{h} / S_{v}\right)$ and external orientation camera parameters $\left(\omega, \phi, \kappa, X_{c}, Y_{c}, Z_{c}\right)$ can be determined from the calibration. $K_{1}$ and $K_{2}$ define the radial lens distortion parameters, and $P_{1}$ and $P_{2}$ define the decentering distortion parameters. 
$S_{h} / S_{v}$ represents the horizontal and vertical pixel spacing ratio for the camera sensor. ${ }^{6}$ An optimization method, originally proposed by Liu $^{7}$ utilizes a single-image camera calibration routine that provides easy on-the-job camera calibrations. This eliminates the need for multiple-image or special laboratory calibrations that are not very well suited for wind tunnel applications. The optimization method involves two interacting procedures that establishes the interior orientation and lens distortion parameters as well as the exterior parameters using a least-squares estimation methodology. ${ }^{6}$ A 3-step calibration plate, with a known 3-D accuracy of 0.001 inch, is used as part of the single-image camera calibration procedure.

\section{VMD Hardware and Software}

The single-camera, single-view approach utilizes a video-rate CCD camera and an accompanying PC to capture and record image sequences through an image acquisition frame grabber board. A parallelprocessing architecture for image acquisition and data processing are used to extract near real-time measurements. ${ }^{5}$ A two-camera VMD measurement system utilizes much of the same hardware packaging (PC, frame grabbers, etc.) as the single-camera system. Two-camera applications are normally used for wind tunnel testing in situations where a single coordinate constraint is not possible. Single camera implementations are usually preferred since camera viewing port access is generally limited, lighting and undesirable glares can be difficult to control for two or more cameras, and tunnel vibrations during testing can cause potential unsynchronized camera jitter that contribute to the uncertainty. However, when target motions are in 3D with no constrained (known) coordinates, multiple camera implementations must be used.

\section{Model Deformation Measurement}

Retro-reflective targets or passive targets are often placed on the main wing element, control surfaces, and a rigid component of the model, such as the fuselage or the base of the wing near the fuselage. This target layout allows for baseline comparisons between wind-off to wind-on deflections at select rigid parts of the model. Digitized video images are captured and immediately processed to determine the spatial locations of targets on a model, which are typically also converted to angular measurements. Angular calibrations based on wind-off polars are used to calibrate the measurement system in terms of angle.

A potential angular bias error exists when using the single-camera, single-view photogrammetric technique for deflected control surfaces in the presence of wing bending. ${ }^{8}$ Without flow the $Y$-coordinate remains constant as the model is pitched whereas with flow the wing encounters bending that causes slight changes in the span-wise location of the targets. This bending causes single-camera photogrammetric angular bias errors, primarily on control surfaces that are experiencing large deflection angles. Whereas the bias errors due to bending for a main wing element will generally be small (less than $0.05^{\circ}$ ); the bias error for control surfaces can exceed $1^{\circ}$. The bias error increases with the amount of bending and the magnitude of the deflection angle of the control surface. A correction procedure based on the invariance of the length between targets can be used to estimate the shift in the $Y$-coordinate used as reference in the photogrammetric computation for control surfaces, thus improving the angular computation. Simulations with exact image plane coordinates and camera parameters indicate that corrections to about $2 \%$ of the error (98\% of error removed) are possible. Controlled wind-off experimental tests with a typical measurement geometry indicate correction to about $15 \%$ of the error is possible ( $85 \%$ of error removed).

\section{Wind Tunnel Facilities}

Aeroelastic deformation measurements have been made in the past at several large production wind tunnel facilities for sting mounted and semi-span wall mounted models ranging from subsonic transports to hypersonic research models. While the primary applications have been at the NTF and the Transonic Dynamics Tunnel (TDT), earlier testing occurred at the 12-Foot Pressure Tunnel at NASA Ames, Langley's Unitary Plan Wind Tunnel (UPWT) and 16-Foot Transonic Tunnel, the 14x22 Foot Subsonic Tunnel, and the Mach $6 \mathrm{CF}_{4}$ Tunnel. More recently, deformation measurements have been made at the 31Inch Mach 10 Tunnel, the 8-Foot High Temperature Wind Tunnel, and the 20-Ft Spin Tunnel.

\section{A. Testing at the National Transonic Facility}

NASA's National Transonic Facility (NTF) wind tunnel is a unique research tool that operates over a large range of temperatures, as low as $-250^{\circ} \mathrm{F}$ using cryogenics (liquid nitrogen), and up to $150^{\circ} \mathrm{F}$ using warm 
air. This variation in temperature along with the ability to vary operating pressures from 15 psia to 130 psia, provide a wide range of Reynolds numbers from 4,000,000/ft to 145,000,000/ft at Mach 0.1 to Mach 1.2. These features offer full-scale Reynolds number testing for scaled models of a wide range of aircraft that are tested in its $8.2 \times 8.2 \times 25$ foot test section.

When conducting a test at NTF, it can be extremely difficult to optimize the lighting between two or more cameras because of limited window port access and limited lighting options in the test section. In addition, models designed for testing at high Reynolds numbers require a mirror-like surface finish, approaching several micro-inches that can potentially produce troublesome glare. Glare can be small enough to be mistaken for additional targets on the model or large enough to wash out the actual targets. Choices of target sorting and identification schemes have been developed to handle some of the issues of glare, but are still not yet versatile enough to completely resolve all of the problems that can be encountered. Developments are underway to further enhance the VMD techniques that are currently in use at the NTF and other wind tunnel facilities.

The wide range of operating temperatures at the NTF tunnel poses major problems for most instrumentation enclosures in or near the test section. For instance, camera packages must be kept warm when the tunnel is operating at cryogenic temperatures and kept cool when at elevated temperatures to ensure proper performance and to reduce the risk of damage. Extreme changes in temperature will cause the model, test section, and the wind tunnel itself to either expand or contract which may introduce bias errors that are very difficult to quantify. Wind-off polar sweeps at each tunnel condition are used as a reference for wing twist computations to partially account for bias errors that may be present. Both windoff and wind-on data are computed from captured images at roughly the same pressure and temperature operating conditions. Model pitch angles obtained from the onboard accelerometer are used to back out the differences between wind-on and wind-off test point pitch angles in order to determine flow-induced wing twist. $^{9}$ VMD targets are typically placed on the main wing element and control surfaces along various chord locations and on a rigid body component of the model such as the fuselage or the base of the wing near the fuselage. These rigid, non-flexing targets are considered reference targets for which the wing twist measurements are based.

Recent VMD tests at NTF included full span and semi-span models such as a 777 semi-span low-speed model, a Blended Wing Body (BWB) transonic model, and the Pathfinder Check Standard wind tunnel models. A 777 semi-span model was tested at the NTF to further understand ground to flight scaling methods and provide data for validation of computational methods. A technique was developed that allowed a single-camera single-view VMD system to independently zoom in on four different chord stations on the leading edge slat along the semi-span of the wing, from an inner most position on the wing to a position out near the wing tip. Each chord station contained four painted and polished targets with two targets on the LE slat and two targets on the main wing element (Figure 1). Repeatable camera alignments were made with a pan-tilt adjustment mount that could accurately reposition the camera from one chord location to another. Camera calibrations were made at each of the four stations, along with saved optimal lighting adjustments for each view. Limited data sets were acquired before failure of the camera pan-tilt adjustment hardware. As a result data was recorded only at the chord location of greatest concern for the remainder of the test. It was determined by the conclusion of the test that only small angular slat deformation (at all conditions) was being recorded that was within the noise level of the VMD system, having a standard deviation of 0.13 degree.

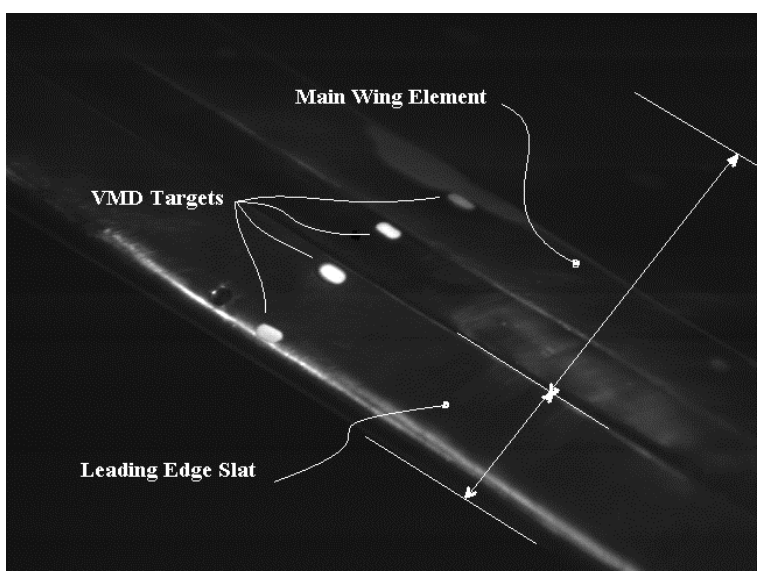

Figure 1. VMD target layout for the 777 semispan model at NTF. 2-targets on the leading edge slat in-line with 2-targets on the main wing element. 
A radical new design concept for future air transportation is the Blended Wing Body (BWB) configuration. The aircraft resembles a flying wing whose fuselage blends with its wings creating a single aerodynamic surface capable of generating more lift and creating less drag. BWB models have made repeated tunnel entries at NTF as part of an on-going research program. VMD measurements produced during these tests have provided valuable model deformation and wing twist data in part to validate design tools for the propulsion/airframe integration for NASA's Ultra Efficient Engine Technology (UEET) Project. Highly polished full-span BWB models are mounted on the circular arc sector in the test section and subjected to a range of pitch angles at transonic flight conditions. Some of the VMD challenges associated with testing this particular model have been excessive glares caused by the relatively flat design of the wing body and the engine nacelles (Figure 2). Reflections from lights, tunnel walls, and engine nacelles often interfere with the VMD targets making them hard to track. Wing twist deflections are measured as aerodynamic loads deform the model. Figure 3 represents a typical data set for angular twist measurements taken by the VMD for the BWB model.
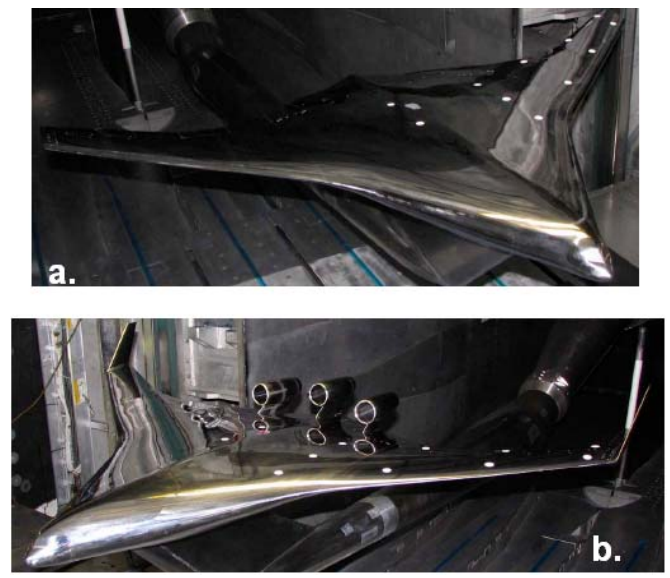

Figure 2. Painted (white dots) VMD targets for the Blended Wing Body (BWB) model.

a.) Model with no engine nacelles.

b.) Model showing reflections from attached engine nacelles.

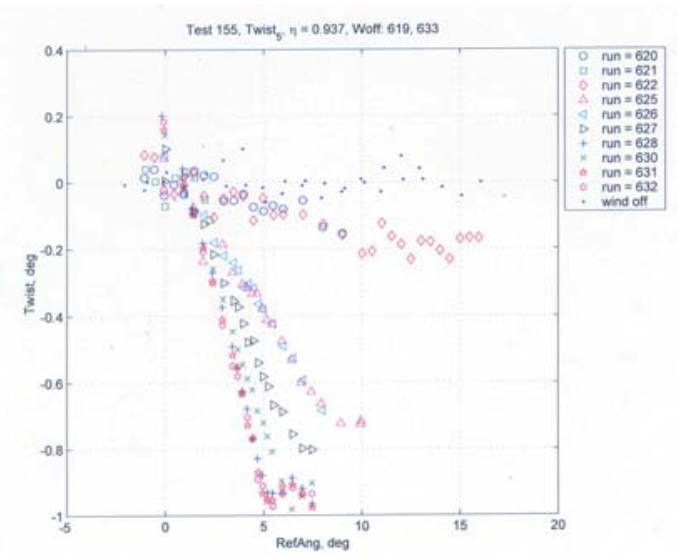

Figure 3. VMD angular twist deflections as a function of model pitch (RefAng) for the BWB model.

NASA's Pathfinder 1 and Half-Scale Pathfinder models are among the first fabricated for testing at the NTF wind tunnel. They were originally designed to demonstrate the feasibility of design and fabrication of models being tested at NTF. Today, they are used on a regular basis as a check standards model for NTF calibration and validation of steady-state flow characteristics and Reynolds number effects. VMD measurements were recently included as part of the data collection procedure for the Pathfinder Check Standards calibration checks performed at the tunnel. Both full-scale and half-scale Pathfinders took part in independent tests designed to endorse the reliability of the measurement capabilities for internal and external customers using the NTF facility.

\section{B. Testing at the Transonic Dynamics Tunnel}

Another unique research tool at NASA is the Transonic Dynamics Tunnel (TDT). This tunnel is predominately used for conducting flutter-clearance and aeroelastic research tests on a national scale for NASA, the DOD, and industry involving fixed-wing, rotary-craft, and launch vehicle concepts. The TDT makes use of sidewall mounted semi-span models, full-span sting mounted models, cable-mounted free flying vehicles, and rotor blade research. The TDT test section measures 15.5 x 15.5 feet and has a continuous-flow, variable-pressure mode of operation that runs in air or heavy gas and operates up to approximately Mach 1.2. The first automated photogrammetric measurements at the TDT Facility occurred in 1994. Recent VMD tests at TDT include an Air Force funded high lift-to-drag flight program and a morphing wing program. 
A two-part joint Air Force, NASA, and industry project, HiLDA (High Lift-to-Drag) / Aerodynamic Efficiency Improvements (AEI) Program, has been tested at TDT. The first series of tests involved aeroservoelastic wind tunnel testing in which the model was subjected to sinusoidal vertical gust excitation at constant and continuous varying frequencies to illustrate potential gust-load-alleviation capabilities. ${ }^{10}$ The HiLDA Semi-Span Wing model (Figure 4) design consists of 20 individually segmented chord sections positioned in a streamwise direction along the wing. This semi-span model has one leading edge control surface near the wing tip and four aileron control surfaces. VMD measurements (Figure 5) were

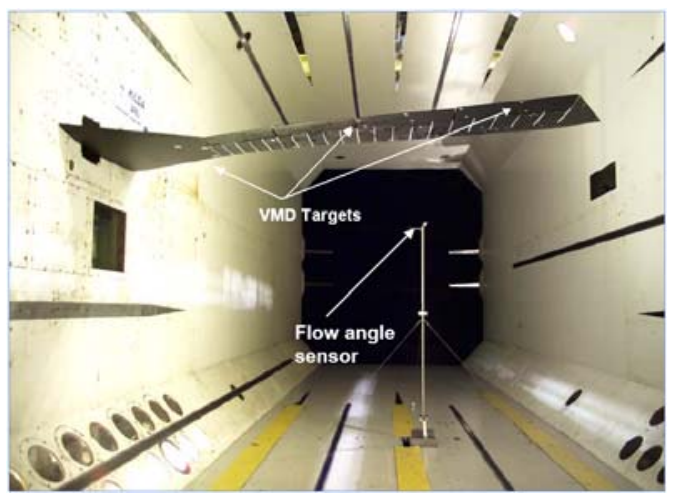

Figure 4. HiLDA model in the TDT test section, looking upstream. ${ }^{8}$

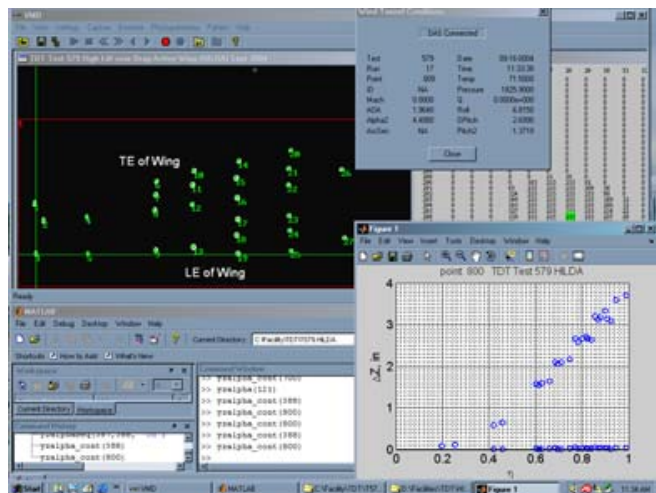

Figure 5. VMD user display showing 26 HiLDA model targets being tracked, Data Acquisition System window display, and a static data plot of $\Delta \mathrm{Z}$ vs. $\eta(\mathrm{Y} / \mathrm{b} / 2)$.

acquired during the full duration of the test and used for analysis of analytical models. Figure 6 shows a typical dynamic data plot of 26 targets at 60 samples per second. For the second part of this test program the HiLDA model was mounted to the tunnel wall in a unique manner. A sidewall mount provided multiple degrees of freedom enabling it to fly freely while still attached to the wall. Model movement at

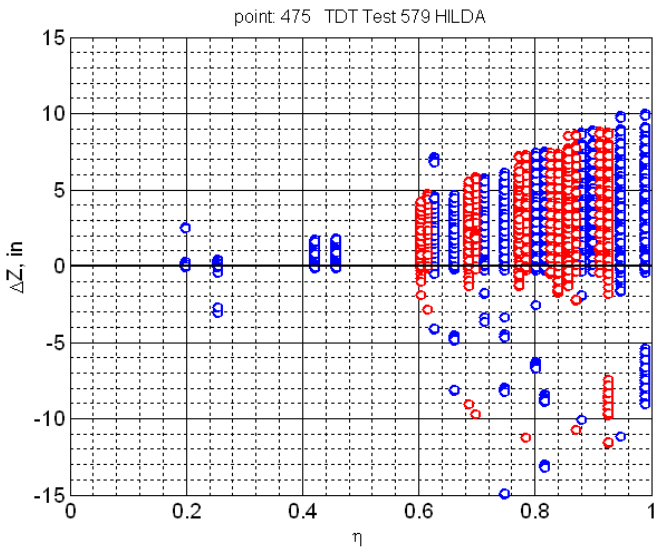

Figure 6. Typical dynamic data plot of $\Delta \mathrm{Z}$ vs. $\eta(Y / b / 2)$ for a Test Point condition at $60 \mathrm{~Hz}$ sampling for 1 second. the wing root had up to 24 inches of vertical travel, 12 inches of fore/aft travel, and approximately -10 to +15 degrees AoA, allowing better simulation of SensorCraft ${ }^{11}$ rigid body motion. Some VMD challenges encountered with this test included a changing wing perspective when the vertical motion of the freeflying wing caused targets to disappear and then reappear on the image plane. Also, steep pitch angles, moving ailerons, and LE control surfaces cause target views to be lost depending on the viewing angle of the camera. Two independent single-camera, single-view VMD systems were employed to help alleviate some of these problems. One system was dedicated to measuring the inner half of the wing starting at the wing root while the other system was

dedicated to the outer half ending with the wing tip. This allowed for longer focal length lenses, which help improve measurement precision. Unfortunately, VMD setup time was limited due to model installation overruns and inaccessibility to the test section, so that no usable data was obtained. Problems also existed with the model's free flying performance. These technical issues are being reviewed and valuable information and lessons learned concerning the use of VMD for this type of testing will be applied when the model returns for further testing at a later date. 

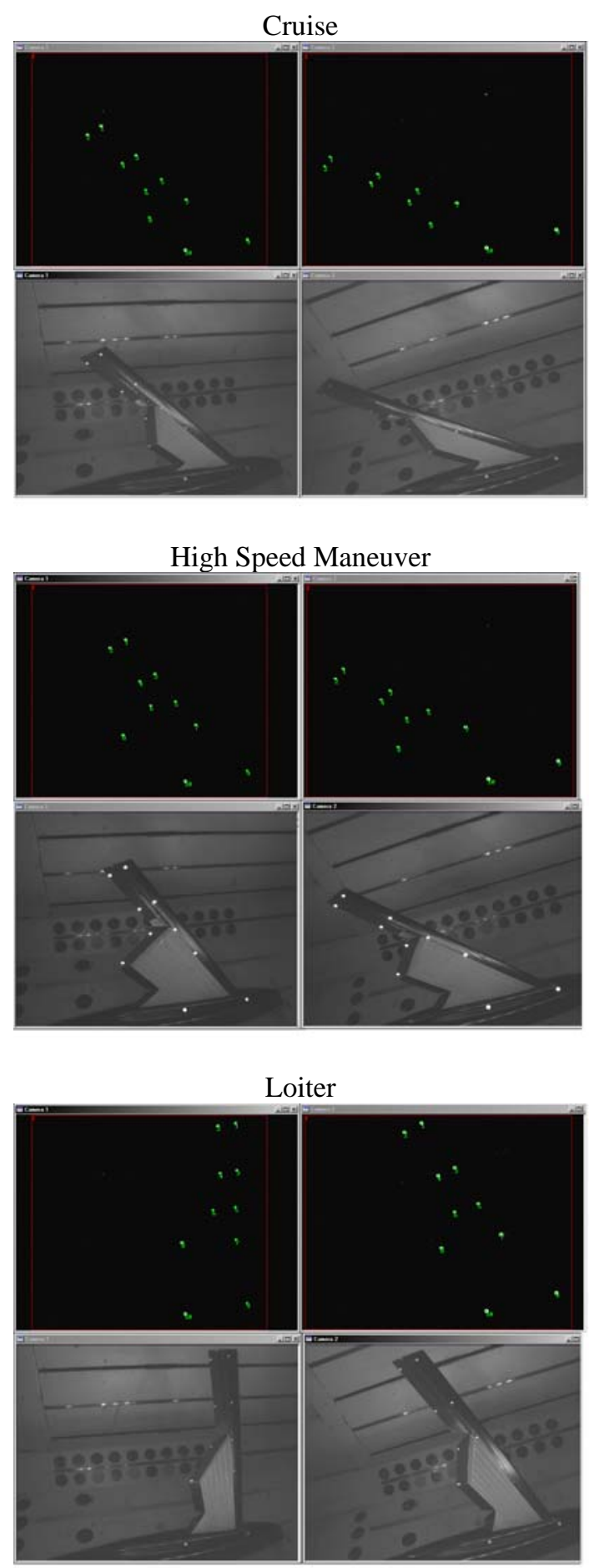

Figure 7. Wind tunnel testing of morphing wing through three different configurations as seen by the Videogrammetric Model Deformation (VMD) measurement system.
New aircraft designs that tend to mimic nature are emerging through advanced morphing technologies. The new capabilities and expectations will entail significant changes in vehicle shape through metamorphosis in a simple, energy efficient manner requiring extensive versatility and resilience. ${ }^{12}$ Videogrammetric measurement techniques are being adapted to the challenges presented by the Morphing Program for wind tunnel testing. The first applications of VMD involving morphing models were with the Smart Wing Program ${ }^{13}$ at TDT, which introduced some early wing reshaping developments with ailerons that mimicked bird-like wing behavior. The latest round of morphing tests conducted at TDT encompassed complex models that dramatically change wing shape while flying at nearly the speed of sound. New measurement challenges include changing wing geometries and models that fold in on the fuselage or simultaneously expand and shrink in chord and span directions. Single-camera measurement applications cannot handle these types of tests so currently a twocamera VMD system is being used. Figure 7 shows two-camera images of a morphing model undergoing a range of wing motion while in the tunnel test section. It is important to maintain stable target sorting conditions when the model is morphing or changing in AoA. A top-down target sorting algorithm is used that keeps the entire target numbering order in a proper sequence provided any 2 targets cannot change position with respect to the horizontal. If this happens or if a target is lost, all of the targets will shift by a count of one to compensate for the missing target. Proper planning and camera placement prevents this from occurring. The data in Figure 8 illustrates static wing deflection at all the target locations for a single Test Point Number, where 30 samples of captured data are averaged over a 1 second time span.

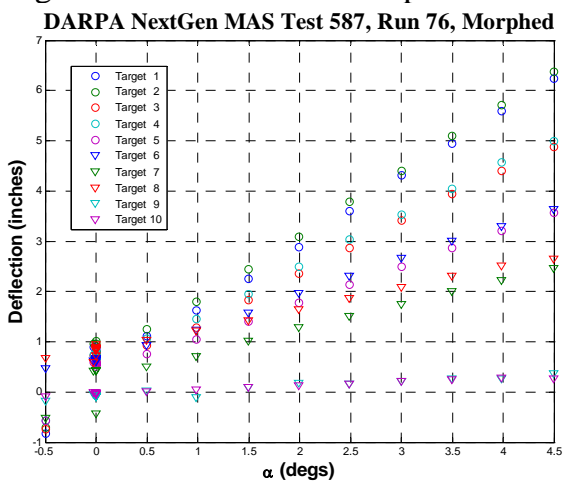

Figure 8. Typical wing deflection data for one of the morphed wing configurations. 
When the TDT is operating in heavy gas mode at reduced atmospheric pressures, a substantial heat buildup occurs in the VMD light sources that must be located relatively close to the test section's lights. Thermal cutoffs on the VMD lights cause them to cycle on and off during testing. Several light systems have been installed for a single camera that utilize staggered cutoff cycles in an attempt to alleviate periods of no lights and loss of data with only partial success. Recently, LED light rings around VMD cameras have been found to produce sufficient light for the retro-reflective targets and do not suffer from the thermal cutoff problem, and should eliminate the light loss problem for future tests.

\section{Testing at the Hypersonic Wind Tunnels}

Videogrametry has been employed at NASA's 31-Inch Mach 10 and Eight-Foot High Temperature wind tunnels. The 31-Inch facility utilizes a closed 31 x 31 inch test section that operates in dry air at stagnation pressures and temperature of $150-1450 \mathrm{psia}$ and $1850^{\circ}$ Rankin with a maximum run time of $120 \mathrm{sec}$. The Eight-Foot High Temperature Wind Tunnel has an open-jet test section that is 8-feet in diameter and 12feet long. It is a combustion-heated hypersonic blowdown-to-atmosphere tunnel that operates at speeds of 4, 5, and 7 Mach at simulated altitudes of 50,000 to 120,000 feet. Each of these tunnels uses hydraulic injection support mechanisms that position models into an active flow field and provides angle-of-attack pitch capabilities when testing.

Evaluations have been made of both single-camera and multi-camera videogrammetry applications at the 31-Inch Mach 10 and Eight-Foot High Temperature facilities. Model positioning variables often include pitch, roll, and yaw during most testing which is undesirable for single-camera techniques. Therefore, a two-camera setup was initiated that can account for the three degrees of freedom. Optical access at hypersonic facilities can be exceptionally challenging due to limited window ports. At the Mach 10 tunnel, a structural camera mounting support was assembled on top of the facility that securely held the positions of two cameras. Studies were conducted to investigate what effects the tunnel vibration had on the measurement precision. No noticeable camera vibrations or shifts in controlled target positions were noted. Data sets consisting of 80 second tunnel runs, have been obtained at the Mach 10 tunnel with a two-camera videogrammetric measurement system. ${ }^{14}$

In addition, the first use of a VMD measurement system at the Eight-Foot High Temperature wind tunnel has been attempted on a hypersonic scramjet propulsion model. Some challenges associated with this facility are the viewing port accessibility and the high temperature open-jet test section. A single-camera VMD technique must be used at this facility because of limited space for test equipment. Therefore, difficult challenges are encountered when determining proper camera position, optimizing tunnel lighting, and establishing camera calibrations. The layout of the tunnel test section makes it extremely difficult to reference the camera calibration block to the model and the tunnel coordinate system when performing a camera resection, which establishes a relationship between the tunnel coordinates and the camera. Procedures are being worked out to overcome some of these difficulties. For instance, two laser-light levels may be used to orient the calibration block orthogonal to the floor and walls of the test section. An accurate reference distance between the cal block and the model would then be needed. The complication with this is the model, mounted to the hydraulic injection arm, is not accessible when the tunnel is not in operation. Details such as this are still being worked. When the tunnel is in operational status the VMD measurement system is currently being used to determine model deformation along the leading edge cowl flap of a scramjet fuel-injection inlet when exposed to the high temperatures associated with the open jet. A variety of experimental targets made from different materials have been tested for survivability in the high temperature open-jet test section. Effective targets, capable of being used for VMD measurements are ones that maintain a high contrast between the targets and the base material that the targets are attached to. In most cases the open-jet in the test section burned away the targets leaving little available contrast to distinguish their presence. White high temperature paint seems to be the most effective material at this time for target proven endurance and has been applied to the model for the next testing opportunity.

\section{Testing at the 20-Foot Vertical Spin Tunnel}

The 20-Foot Vertical Spin Tunnel is a unique vertically erect wind tunnel that operates at atmospheric conditions. The test section stands 25 -feet tall and has an approximate 20 -foot diameter throat. Variable speed, fixed-pitched fan blades are used to reach test section velocities of $85 \mathrm{ft} / \mathrm{sec}$ thus promoting rapidly 
changing fan speed flow accelerations in order to maintain equilibrium for dynamically scaled free-flying models. During normal operating conditions models are hand launched into the wind flow with a prerotation in order to study aerodynamic spin characteristics.

A state-of-the-art 8-camera commercial videogrammetric measurement system is currently in service at the 20-Foot Vertical Spin Tunnel. The COTS package has been designed to meet the needs of this unique wind tunnel and is performing well during initial checkout testing.

\section{A. In-Flight Videogrammetric Measurements}

\section{Other Videogrammetric Applications}

A videogrammetry technique (utilizing an onboard video camera) normally used for wind tunnel testing was adapted for aeroelastic measurements during flight-testing. The method was tested on the wing of an F/A-18 research aircraft of the Active Aeroelastic Wing (AAW) Program flying at Mach 0.95 at an altitude of 15,000 ft. Spanwise wing deflection and wing tip time histories of aerodynamically induced changes in twist and deflection through a maneuver were extracted successfully from on-board images during flight. Flight tests have been conducted that utilized different on-board wing deformation measurement capabilities. A specially fabricated viewing pod was installed on the top of the fuselage that supported an electro-optical Flight Deflection Measurement System (FDMS), a flight-qualified video camera, and a 3mega-pixel SLR digital still camera. Each of these packages was used in support of the wing twist and deflection measurements for the left wing, as seen through the viewing pod. Methodologies for flight wing measurement techniques have been compared to that of tunnel wing measurement techniques. Figure 9 illustrates the differences in camera geometry for wind tunnel testing, with a camera mounted on the test section wall, and in-flight testing, with a camera mounted on the fuselage. The measurement of wing deformation during flight testing is closer to a differential measurement, can make better use of the available image area, and is affected less by rigid body motion because the camera is on the fuselage which is considered a rigid body. The dynamics of a wind tunnel model can be more complicated than for in-flight testing because of a necessity to accommodate and account for rigid body motion. For example, the camera on the fuselage of an aircraft moves in concert with the fuselage and any dynamics common to the fuselage and wing tend to cancel. Cameras are not normally mounted on the fuselage of wind tunnel models primarily due to size constraints.

The adaptation and application of videogrammetric model deformation measurement techniques to aircraft in flight are still in early stages of development. Actual in-flight recordings taken aboard the F/A-18 aircraft illustrated useful measurements are possible even when a low-resolution video camera, normally used for surveillance, is used to acquire images. Comparisons between measurements made with the FDMS and the video surveillance camera were made while on the runway and during several flight maneuvers. The estimated precision for the image coordinate locations during flight was about one pixel due to the low-resolution camera and large data scatter. It is estimated that the precision could be reduced by about a factor of 10 if better-defined, higher-contrast targets were used. ${ }^{15}$ 


\section{B. Aerodynamic Loads Based on Optical Elastic Deformation Measurements}

A videogrammetric technique for determining aerodynamic loads based on optical elastic deformation measurements has been developed and laboratory tested at NASA. The methodology is based on beam deformation theory. Data reduction methods are used to extract the normal force and pitching moment from beam deformation data. Axial force can be obtained by measuring the axial translational motion of a movable shaft in a spring/bearing device. A two-camera VMD system is used for the optical deformation measurements and the data reduction models for extracting the normal force and pitching moment utilize either the local displacement and slope change, or the global beam deformation profile. The optical method has the capability of remote non-contact measurement that can be readily integrated with the optical methods of model attitude measurement and used as a potential alternative in certain cases where strain gauge methods cannot be easily applied during wind tunnel testing. ${ }^{16}$ Wind tunnel flutter tests have been performed on three thin cantilever plates in a low speed wind tunnel to estimate aerodynamic forces taken from videogrammetric deformation measurement data. Analyzed results indicate successful recovery of unsteady aerodynamic forces is possible, with potential applications for wind tunnel or aircraft testing. ${ }^{17}$

\section{Ultra-Lightweight and Inflatable Space Structures}

Videogrammetric measurement techniques are often used for the structural characterization of space structures. Ground based studies in support of advanced materials for large, ultra-lightweight deployable and inflatable space columns, solar arrays, large antennas, and solar sails have been performed at NASA. Photogrammetry has been used in many of these studies to provide shape and dynamics measurements. Some advanced materials' objectives might include large ultra-lightweight flexible membranes for solar sails that must be durable and consist of little mass or inflatable structures that can be rigidized when put into service. Experimental and analytical investigations are necessary to characterize structural performances for future applications outside the Earth's atmosphere. These photogrammetric measurement applications have included techniques such as laser-induced fluorescence ${ }^{18}$, dot projection ${ }^{19}$, and object-toimage plane scaling. ${ }^{20}$ The success of these techniques has demonstrated the ability of photogrammetric measurements to provide extremely useful information even when faced with measurement challenges associated with reflective materials, extremely large test articles, and limited test budgets.

\section{Crew Exploration Vehicle (CEV) Soil Characterization}

A technique using object-to-image plane scaling to measure the dynamic impact for CEV model drop testing is currently underway. The next generation spacecraft, to replace the aging Space Shuttle, calls for a manned spacecraft program intended to carry human crews from Earth into space and back. Studies are being conducted to determine the feasibility of landing the craft in the desert rather than the ocean like during the Apollo Program. A low cost optical technique that is relatively simple to implement has been developed that utilizes image processing and image scaling to measure the spatial positioning of discrete targets on the CEV models, as they impact the ground. High-speed cameras (1000 frames per second) with good image resolution are being used to track the targets from different viewing perspectives. Calculations are made for pitch and yaw angle changes and vertical and horizontal velocities of the CEV and the data obtained during the ongoing testing have helped validate computational design geometries.

\section{Future Development}

The VMD measurement systems developed at NASA for wind tunnel applications are commonly used at the NTF and TDT facilities. Other wind tunnel and non-wind tunnel VMD measurement requests are often made and the system performances for these requests generally prove to be admirable. As test conditions become more challenging and system requirements increase, so do the demands for additional performance. Some recent measurement challenges include extreme heat and poor visual access during hypersonic wind tunnel testing, rotorcraft blade measurements, parachute deployment and descent, large space structures and solar sails, and full-scale crash testing. Multi-camera, multi-image, and multi-ray intersection geometry is an effective means of ensuring enhanced measurement accuracy. There are reputable commercially available photogrammetric measurement systems on the market that can fulfill the demands for many of these challenges. Unfortunately, many aerospace applications do not allow the luxury of multiple cameras, particularly in the wind tunnels. Therefore, the robustness and usability of the single-camera, single view and two-camera VMD measurement systems have undergone continuous improvement at NASA through the years and will need to continue to do so in order to ensure data quality, applicability, usability of the 
measurement systems, and to ensure technological leadership. Applications of high-speed, high-resolution camera support, extended target recognition, and robust camera calibration methodologies are some of the techniques being developed, which leverage state-of-the-art technology to meet new measurement challenges.

The current VMD measurement techniques commonly used in NASA's wind tunnels typically measure the change in static wing twist and static and dynamic wing deflections due to aerodynamic loading. On occasion, model mishaps can occur. A leading edge control surface or aileron on a wing can loosen and begin to move and eventually even break away from a model, or a flutter model can exceed its predetermined bending limits, endanger the safety of the model, and jeopardize the entire test. Therefore, some tunnel tests, particularly at the TDT facility, require specific dynamic measurements of wing deflection. The results are used to aid near real-time decisions regarding model integrity (model safety) before continuing to a new tunnel Run-condition. The VMD measurement system has been used to monitor the structural health for some of the models at TDT (Figure 10) to help determine and avoid many potential model safety issues before they occur. Software enhancement in the area of model safety involving VMD measurement will be assessed for future development. ${ }^{21}$

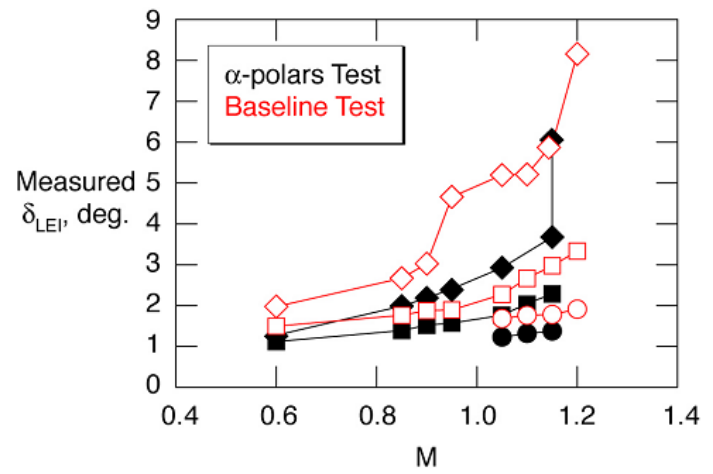

Figure 10. Leading edge control surface angular deflection as a function of Mach for increasing dynamic pressure (circle - low q, square- medium q, diamond - high q). Solid symbols indicate testing sequence just before model failed at high $q$ (solid diamonds).

\section{Conclusion}

The videogrammetric technique discussed in this paper has proven to be useful for non-intrusive measurement of wind tunnel model attitude, wing twist, deformation, and displacement. Single-camera, single-view and two-camera VMD measurement accomplishments have helped provide a better understanding of the aeroelastic properties for a variety of model designs at some major wind tunnel facilities. VMD systems are not just wind tunnel specific; other successful aerospace measurement applications have also been made for a variety of ground and flight tests. Future VMD enhancements should increase system robustness through faster and more capable videogrammetric applications and techniques. The implementation of high-speed, high-resolution camera support and improved target recognition and registration will significantly contribute to increasingly robust and more routine use of VMD in the future.

\section{References}

1. Burner, A. W. and Liu, Tianshu, "Videogrammetric Model Deformation Measurement Technique”, Journal of Aircraft, vol. 38, No. 4, July-August, 2001, pp. 745-754.

2. Fleming, G. A., Soto, H. L., South, B. W., and Bartram, S. M., "Advances in Projection Moire Interferometry Development for Large Wind Tunnel Applications", SAE Paper No. 1999-01-5598, Presented at the SAE World Aviation Congress, San Francisco, CA, October 19-21, 1999.

3. G.A. Fleming, S.M. Bartram, M.R. Waszak, L.N. Jenkins, "Projection Moiré Interferometry Measurements of Micro Air Vehicle Wings”, SPIE International Symposium on Optical Science and Technology, San Diego, CA July 29-August 3, 2001.

4. A. W. Burner, G. A. Fleming and J. C. Hoppe, “Comparison of Three Optical Methods for Measuring Model Deformation”, 38th AIAA Aerospace Sciences Meeting and Exhibit, Reno, Nevada, AIAA 2000-0835, January 10-13, 2000. 
5. Sharon S. Graves and Alpheus W. Burner, "Development of an Intelligent Videogrammetric Wind Tunnel Measurement System”, SPIE's 46th Annual Meeting---The International Symposium on Optical Science and Technology, San Diego, California, July 29-August 3, 2001.

6. Wolf, Paul R., Dewitt, Bon A., "Elements of Photogrammetry”, McGraw-Hill, New York, 2000.

7. Tianshu Liu, L. N. Cattafesta III, and R. H. Radeztsky, "Photogrammetry Applied to Wind Tunnel Testing”, AIAA Journal, Vol. 38, No. 6, June 2000, pp. 964-971.

8. Alpheus W. Burner and Danny A. Barrows, "Error Due to Wing Bending in Single-Camera Photogrammetric Technique”, NASA TM 2005-213254, May 2005.

9. Alpheus W. Burner, Richard A. Wahls and William K. Goad, "Wing Twist Measurements at the National Transonic Facility”, NASA TM 1996-110229, February 1996.

10. Raymond G. Kvaternik, Kenneth W. Eure, and Jer-Nan Juang, "Exploratory Studies in Generalized Predictive Control for Active Gust Load Alleviation”, NASA TM 2006-214296, April 2006.

11. Vartio, E.; Shimko, A.; Tilmann, C. P.; and Frick, P. M.: Structural Model Control and Gust Load Alleviation for a SensorCraft Concept. Presented at 46th AIAA/ASME/ASCE/AHS/ASC Structures, Structural Dynamics, and Materials Conference, Austin, TX, April 2005. Paper No. AIAA-2005-1946.

12. Anna-Maria R. McGowan and Martin R. Waszak, "NASA’s Morphing Project Research Summaries in Fiscal Year 2002”, NASA TM 2005-213266, February 2005.

13. Jennifer P. Florance, Alpheus W. Burner, Gary A. Fleming, Craig A. Hunter, and Sharon S. Graves, "Contributions of the NASA Langley Research Center to the DARPA/ AFRL/ NASA/ Northrop Grumman Smart Wing Program”, AIAA Dynamics Specialists Conference, Norfolk, Virginia, AIAA 2003-1961, April 9-10, 2003.

14. Thomas W. Jones, Charles B. Lunsford, and Sharon S. Graves, "Design and Development of a Real-Time Model Attitude Measurement System for Hypersonic Facilities”, 43rd AIAA Aerospace Sciences Meeting, Reno, Nevada, AIAA-2005-1411, 10-13 January 2005.

15. Alpheus W. Burner, William A. Lokos, and Danny A. Barrows, “Aeroelastic Deformation: Adaptation of Wind Tunnel Measurement Concepts to Full-Scale Vehicle Flight Testing”, Symposium on Flow Induced Unsteady Loads and the Impact on Military Applications, Budapest, Hungary, RTO/AVT-123, April 25-29, 2005.

16. Liu, T., Barrows, D. A., Burner, A. W., and Rhew, R. D., "Determining Aerodynamic Loads Based on Optical Deformation Measurements”, AIAA J. Vol. 40, No. 6, 2002, pp. 1105-1112.

17. John P. Roy, Tianshu Liu, and Colin P. Britcher, "Extracting Dynamic Loads from Optical Deformation Measurements", 47th AIAA/ASME/ASCE/AHS/ASC Structures, Structural Dynamics and Materials Conference, Newport, Rhode Island, AIAA 2006-2187, 1-4 May 2006.

18. Adrian A. Dorrington, Thomas W. Jones, Paul M. Danehy, and Richard S. Pappa, "Laser InducedFluorescence Photogrammetry for Dynamic Characterization of Membrane Structures”, AIAA Journal. Vol. 42, No. 10, October 2004.

19. Pappa Richard S., Black Jonathan T., Blandino Joseph R., Jones Thomas W., Danehy Paul M., Dorrington Adrian A., "Dot-projection photogrammetry and videogrammetry of Gossamer space structures", Journal of Spacecraft and Rockets, Vol. 40, No. 6, November-December 2003.

20. Danny A. Barrows, Judith J. Watson, Alpheus W. Burner, James E. Phelps, “Optical Measurement Technique for Space Column Characterization”, 24th AIAA Aerodynamic Measurement Technology and Ground Testing Conference, Portland, Oregon, AIAA 2004-2185, 28 June - 1 July 2004.

21. Charles V. Spain, Jennifer Heeg, Thomas G. Ivanco, Danny A. Barrows, James R. Florance, Alpheus W. Burner, Joshua DeMoss, Peter S. Lively, "Assessing Videogrammetry for Static Aeroelastic Testing of a Wind-Tunnel Model”, 45th AIAA/ASME/ASCE/AHS/ASC Structures, Structural Dynamics \& Materials Conference, Palm Springs, CA, AIAA 2004-1677, 19-22 April 2004. 